\title{
Design a High Transmission FSS Structure for THz Applications
}

\author{
Muaad Hussein ${ }^{1}$, Haider Alrudainy ${ }^{2}$ \\ \{muaad.hussein@stu.edu.iq ${ }^{1}$,h.m.a.alrudainy@stu.edu.iq $\left.{ }^{2}\right\}$
}

Electrical Power Techniques Engineering, Southern Technical University, Basra, Iraq ${ }^{1,2}$

\begin{abstract}
A unique frequency selective surface (FSS) structure was proposed as a terahertz bandpass filter in this study. The proposed structure is simple to make, has a high transmission of up to 96 percent, a wide bandwidth, and excellent band-edge transitions at $\mathrm{THz}$ frequencies. Based on an investigation of the FSS structure geometry configuration and the distribution of the electric field in it, a theoretical equivalent circuit model is presented to characterise the structure. It has been demonstrated that the comparable circuit model's outputs are in good agreement with numerical simulation tools. The filter is simulated using CST Microwave Studio's frequency selective surface (FSS). To simulate the equivalent circuit, Advanced Design Systems (ADS) is used..
\end{abstract}

Keywords: THz, mesh, FSS, filter, equivalent circuit.

\section{Introduction}

Terahertz (THz) is a frequency range that falls between microwave and optical frequencies. In open space, the emitted signal at $1 \mathrm{THz}$ has a wavelength of $300 \mathrm{~m} . \mathrm{THz}$ systems have been introduced as a brilliant wireless interface approach in recent years. $\mathrm{THz}$ systems are used for medical imaging, $\mathrm{THz}$ spectroscopy, security scanning, high-speed wireless communication, and remote sensing [1-2]. THz bandpass filter could be realized as a combination of frequency-selective surfaces (FSS) comprising arrays of metal print on the front side or both sides a dielectric.

Metal mesh and related structures are suitable elements for the construction of THz filters. The fundamental frequency selective surfaces are classified into the bands top patch type and the bandpass mesh type, as shown in Fig 1. The representation of the THz properties of the grids by an equivalent circuit is very useful to apply the well-developed transmission line theory to calculate $\mathrm{THz}$ filters consisting of two or more grids [3-6]. The equivalent circuits for a band stop FSS type and bandpass FSS type are shown in Fig 2. The grids consist of a thin layer of metal and are supported by a dielectric film. A cross dipole is the most common bandpass grid shape and unit cell, as depicted in Fig 3. (a). Periodicity P, cross member length d, and crossmember width $\mathrm{W}$ define the geometry. The frequency response of the filter is determined by the slot dimensions. If $\mathrm{P}$ is substantially less than the wavelength, linearly scaling the dimensions $\mathrm{P}, \mathrm{d}$, and $\mathrm{W}$ can alter the filter profile [7-8]. 


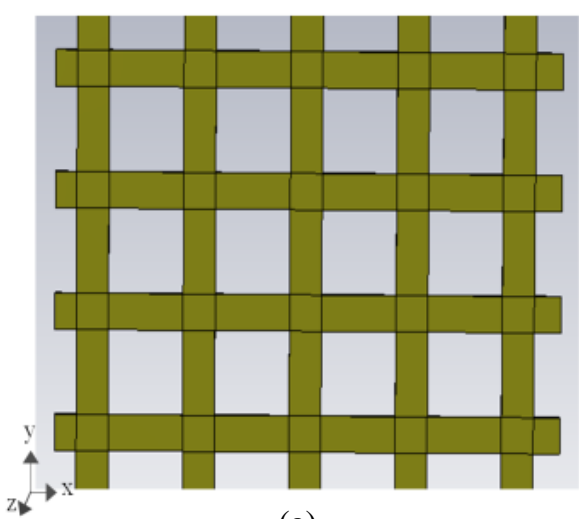

(a)

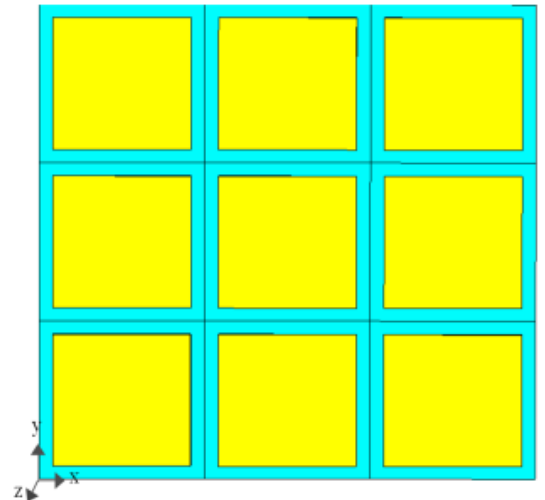

(b)

Fig 1. (a) Bandpass mesh frequency selective surface (Inductive grid) and (b) Band stop patch frequency-selective Surface (capacitive grid)

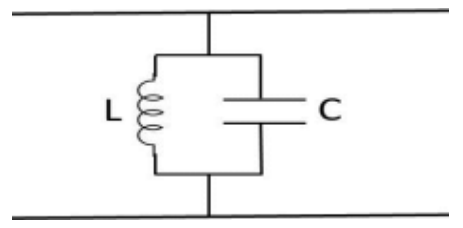

(a)

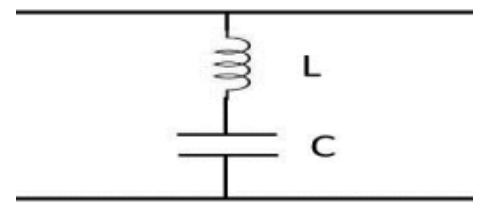

(b)

Fig 2. (a) Equivalent circuit for mesh-type bandpass FSS and (b) Equivalent circuit for patch-type bandstop FSS

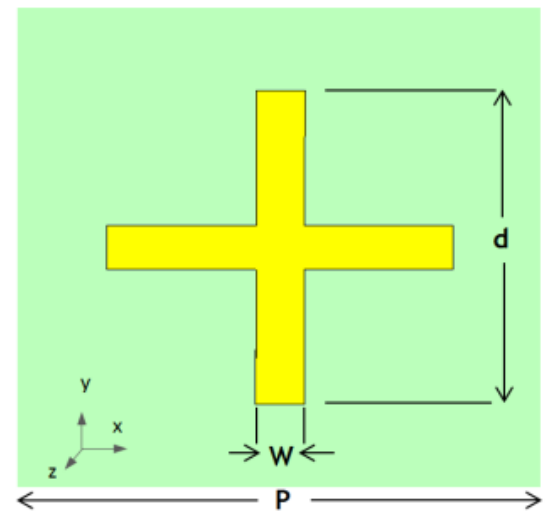

(a)

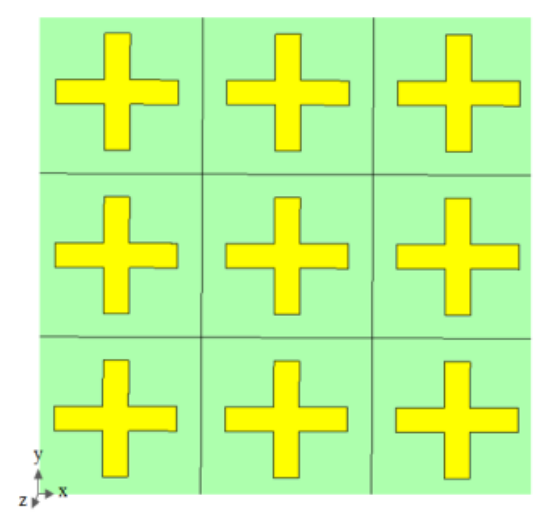

(b)

Fig 3. (a) Cross-shaped filter design parameters lattice constant (P), a width of the cross (W) length of the cross (d), and (b) $3 \times 3$-unit cells of the cross-shaped filter.

Different frequency responses of a filter can be achieved by different FSS element shapes, the conductivity of FSS metal, dielectric constant of the substrate, and incident angle of the signal. 
Figure 3 depicts the cross dipole's $3 \times 3$-unit cells (b). Dual-band and high transmission bandpass filters with up to $90 \%$ transmission have been achieved using dielectric-metaldielectric [9]. The meta-material-based bandpass filter could have an extremely narrow transmission bandwidth [10-11]. The passband's $3 \mathrm{~dB}$ fractional bandwidth is less than $25 \%$ in both cases when utilising two layers of the cross-slot structure and four layers of the wire-plate structure. The transmission amplitude is greater than $80 \%$ [12]. The bandwidth of the filter can be increased by reducing the capacitance effects of the FSS structure [3]. A ring with two splits and two roadways inside the ring is the proposed unit cell structure.

The value of resonant frequency and bandwidth can be adjusted by changing parameters dimensions such as the widths of the ring and the roads, and gap distances as well as road-toroad separation. The advantages of the proposed filter are a high transmission up to $96 \%$, sharp band edge, and a fractional bandwidth of $37 \%$ can be achieved by simulation of the structure. Due to the FSS structure constructs by a metal-dielectric and the smallest dimension at $1.2 \mathrm{THz}$ is $10 \mu \mathrm{m}$, the structure is easy to fabricate. Traditionally, a single metal-dielectricmetal or multi-layer structure is needed to achieve comparable performance.

\section{Filter design and simulation}

The filters are designed to pass $0.4 \mathrm{THz}$ with the least amount of loss. The proposed resonator is constructed by printing a metal structure on one side of a dielectric substrate. The structure was created with the goal of achieving the lowest possible mutual capacitance between unit cells. Copper is the metal, and it has a thickness of $0.15 \mu \mathrm{m}$. The substrate is 20 $\mu \mathrm{m}$ thick Mylar with a permittivity of 2.89 . The ring diameter is $100 \mu \mathrm{m}$. The width of the road is $10 \mu \mathrm{m}$, the gap of each split is $10 \mu \mathrm{m}$ and the gap of the centre of ring $\mathrm{G}_{\mathrm{c}}$ is $10 \mu \mathrm{m}$ as shown in Fig. 4. The periodic constant $\mathrm{P}$ is $60 \mu \mathrm{m}$. The grid of unit cells of the proposed filter is shown in Fig. 5. The simulated result of the terahertz bandpass filter is shown in Fig. 6 . The centre frequency is $1.268 \mathrm{THz}$, and the $\mathrm{S}_{11}$ parameter has reached $-28 \mathrm{~dB}$. The filter exhibits high performances, characterized by the high transmission of the $\mathrm{THz}$ wave with a $3 \mathrm{~dB}$ bandwidth of up to $465 \mathrm{GHz}$ and good band edge transition. According to the incident angle of the electric field and electric current distribution, as shown in Fig. 7, the corresponding equivalent circuit model is found as shown in Fig. 8. This simple RLC circuit can represent the frequency response of an inductive frequency selective surface. L/2 represents the parallel of two inductances, $2 \mathrm{C}$ is the gap of ring centre capacitance, and $\mathrm{R} / 2$ represents the losses. The response obtained by the equivalent circuit model is in good agreement with the numerical simulation of the distribution structure. The transmitted power for this equivalent circuit can be obtained using Eq. 1 [4].

$$
T=\frac{R^{2}+Z_{0}^{2} /\left(\frac{w}{w_{0}}-\frac{w}{w_{0}}\right)}{(1+R)^{2}+Z_{0}^{2} /\left(\frac{w}{w_{0}}-\frac{w}{w_{0}}\right)}
$$

where $\mathrm{Z}_{0}$ is the characteristic impedance of the transmission line, and $\mathrm{w}_{0}$ is the resonant frequency. The lumped elements value to achieve this response is capacitance $15.1 \mathrm{fF}$ and inductance $1.03 \mathrm{pH}$ and resistances as shown in Fig. 9. The splits in the circumference of the ring can decrease the capacitive effect between unit cells and neighbours. The incident angle of an electric field cannot induce capacitive effects in the circumference splits of the ring as shown in Fig. 7. The main capacitive effect is achieved by the centre gap $G_{c}$. This can give full 
control of the resonant frequency by changing the centre gap $\left(\mathrm{G}_{\mathrm{c}}\right)$. The resonant frequency is tuned from $1.268 \mathrm{THz}$ to $1.18 \mathrm{THz}$ with 0.370 fraction bandwidth by decreasing the centre gap $\mathrm{G}_{\mathrm{c}}$ from $14 \mu \mathrm{m}$ to $10 \mu \mathrm{m}$.

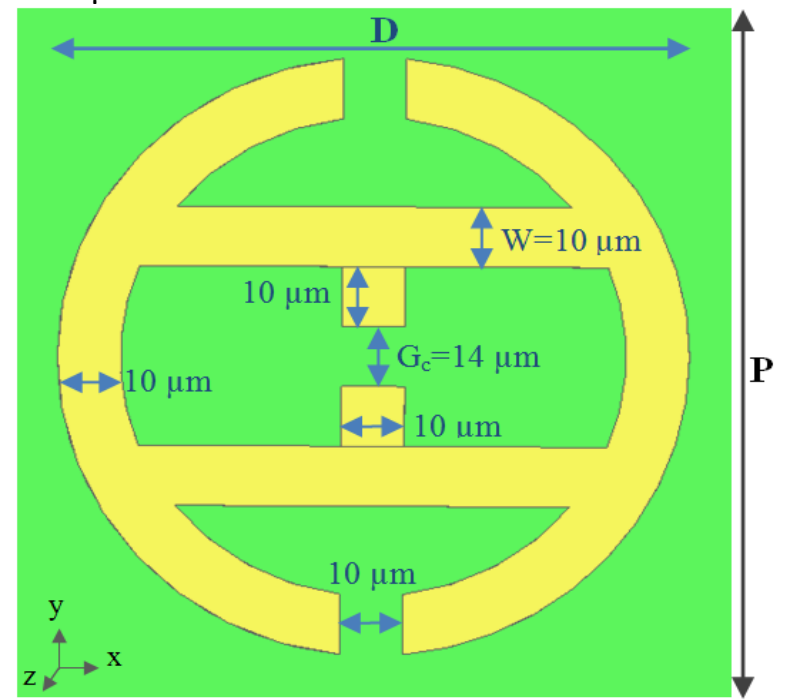

Fig 4. New shaped filter design parameters lattice constant $(\mathrm{P})$, the gap $(\mathrm{g})$, the diameter of the ring (D), and roads width $(\mathrm{W})$.

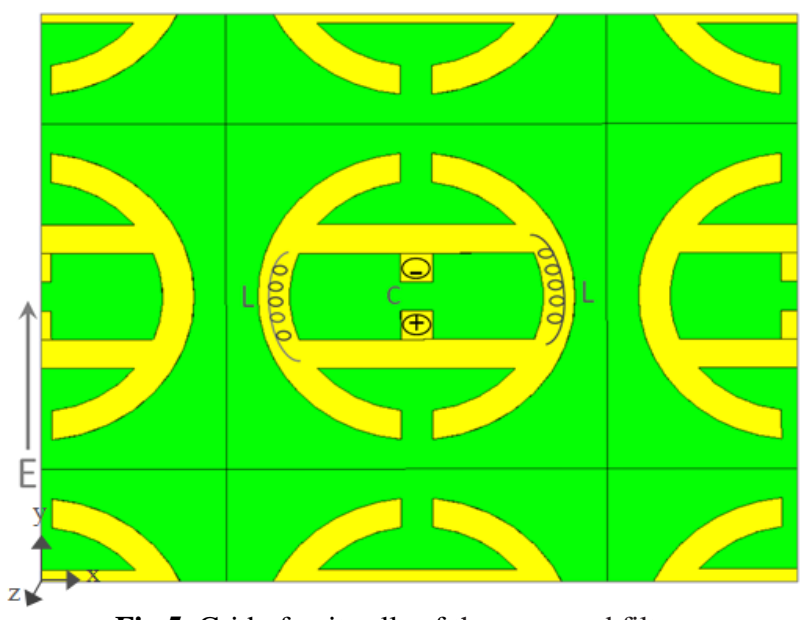

Fig 5. Grid of unit cells of the proposed filter. 


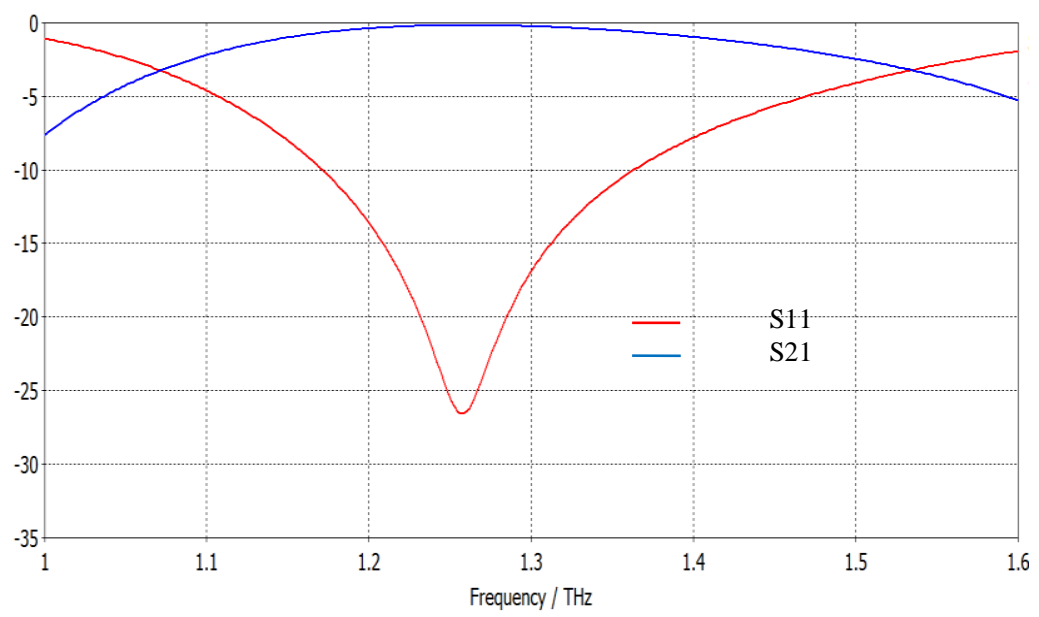

Fig 6. S11 and S21 of proposed bandpass filter by simulated distribution structure in CST.

While the resonant frequency shifted to $1.31 \mathrm{THz}$ when the $\mathrm{G}_{\mathrm{c}}$ became $18 \mu \mathrm{m}$ with 0.385 fraction bandwidth. Fig 10 explains the effect of changing $G_{c}$ on the resonant frequency. The transmitted power for all cases of changing the Gc is $96 \%$. These responses obtained by tuning the value of capacitance (c) in the equivalent circuit to $17.1 \mathrm{fF}$ and $14.3 \mathrm{fF}$, respectively. Changing the wave incident angle can Increase the fraction bandwidth, but at the same time cause a decrease in the transmitted power. Moreover, it can shift resonant frequency towards the lower band as shown in Fig 11.

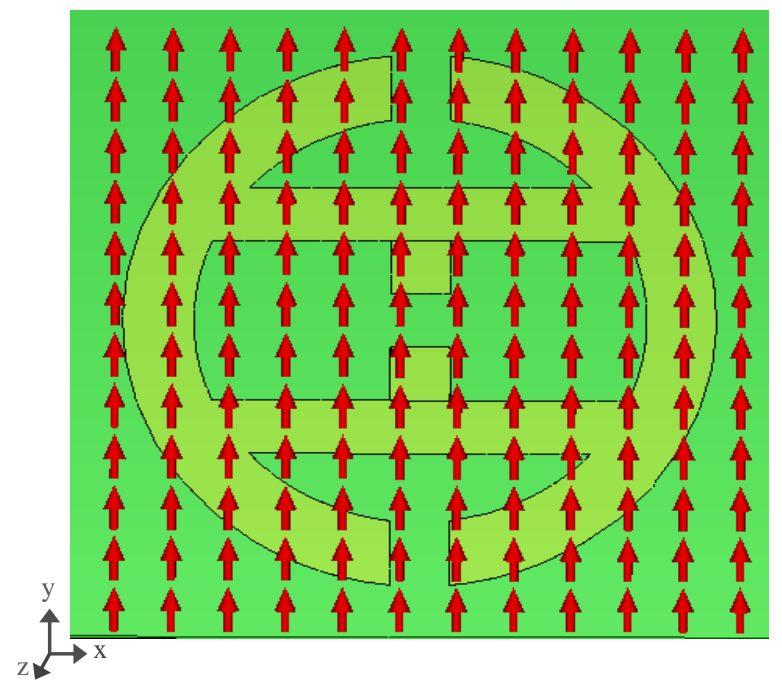

Fig 7. The incident angle of the electric field on the structure is 0 degree. 


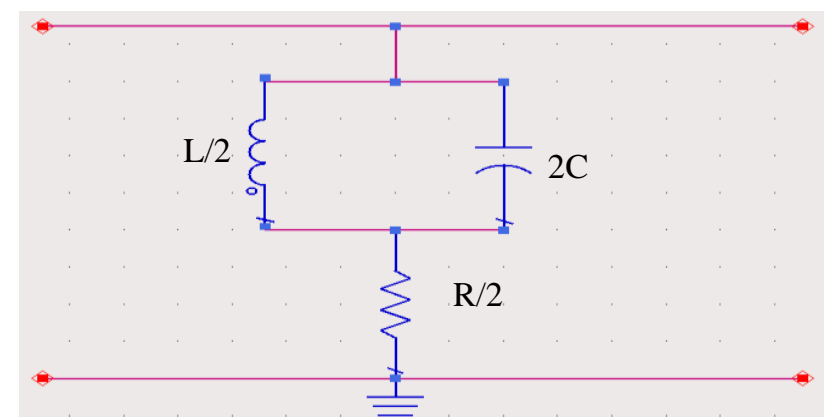

Fig 8. The proposed circuit model.

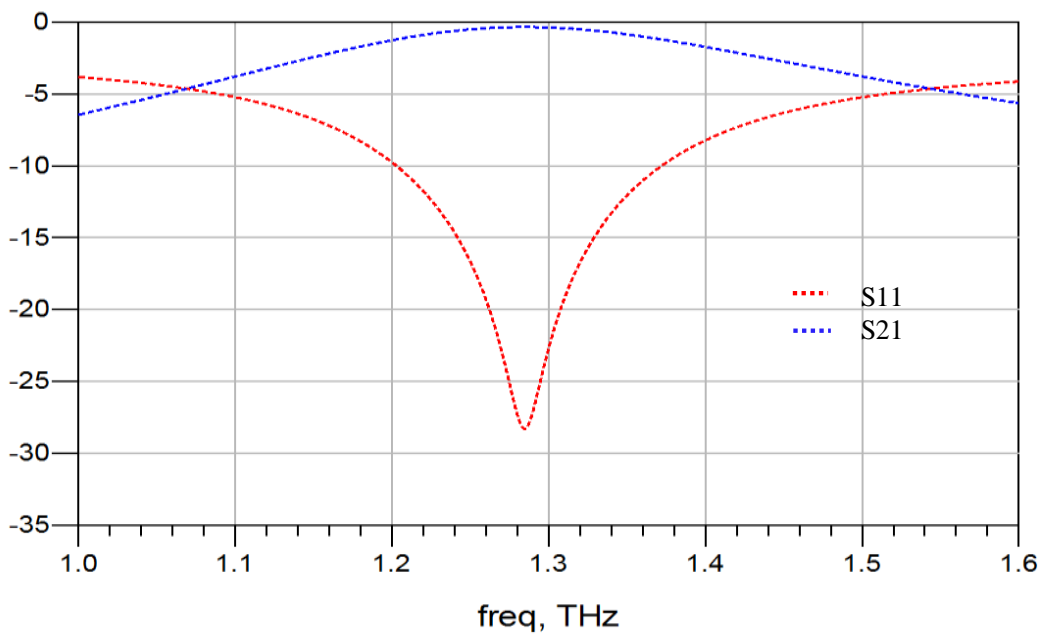

Fig 9. $\mathrm{S} 11$ and $\mathrm{S} 21$ of proposed bandpass filter by the simulated equivalent circuit in ADS.

For instance, changing of wave incident angle to $15^{\circ}$ can shift the resonant frequency to 1.22 $\mathrm{THz}$ and enhance the fraction bandwidth to $40 \%$, while the transmitted power is decreased by $23 \%$. At an incident angle of $40^{\circ}$, the fraction bandwidth is enhanced to be $45 \%$ with an $85 \%$ reduction in transmitted power. After the $45^{\circ}$ angle of the incident wave, the fraction bandwidth starts to decrease, and the transmitted power continues to decrease. It is worth mentioning with changing the incident angle that the resonant frequency is shifting towards the lower frequency. It can use Snell's law to prove that the transmitted power is influenced by changing the incident angle of the wave plane [13]. Thus, a smaller bandwidth is achieved when the $\mathrm{Q}$ factors are higher at the resonant frequency with greater amplitudes. Increasing the permittivity can decrease the bandwidth and resonance frequency of the filter. Reducing the thickness of the substrate can increase the resonance frequency and bandwidth. Table 1 exhibits the metal mesh bandpass filter of different authors [7]. 


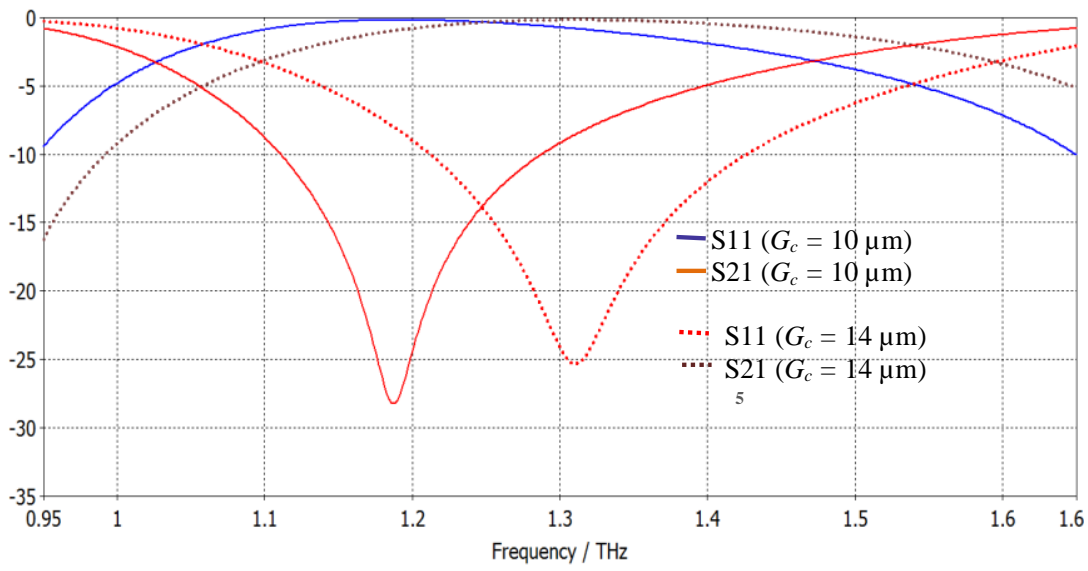

Fig 10. Transmission and reflection of a proposed bandpass filter with $\mathrm{G}_{\mathrm{c}}=12 \mu \mathrm{m} \& 14 \mu \mathrm{m}$ (simulate distribution structure in CST).

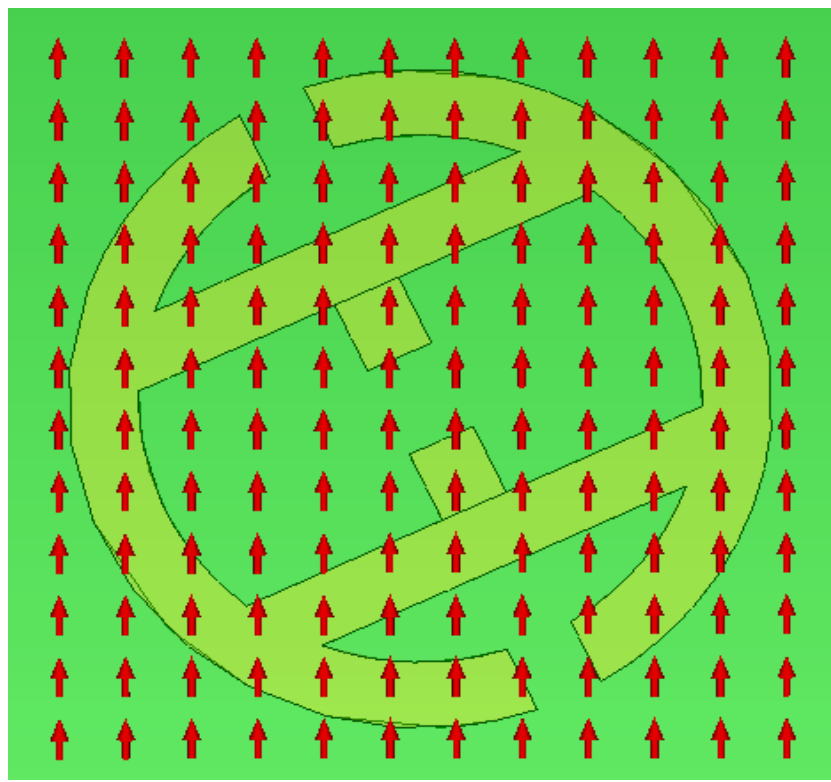

Fig 11. The incident angle of the electric field distribution from 0 degree. 


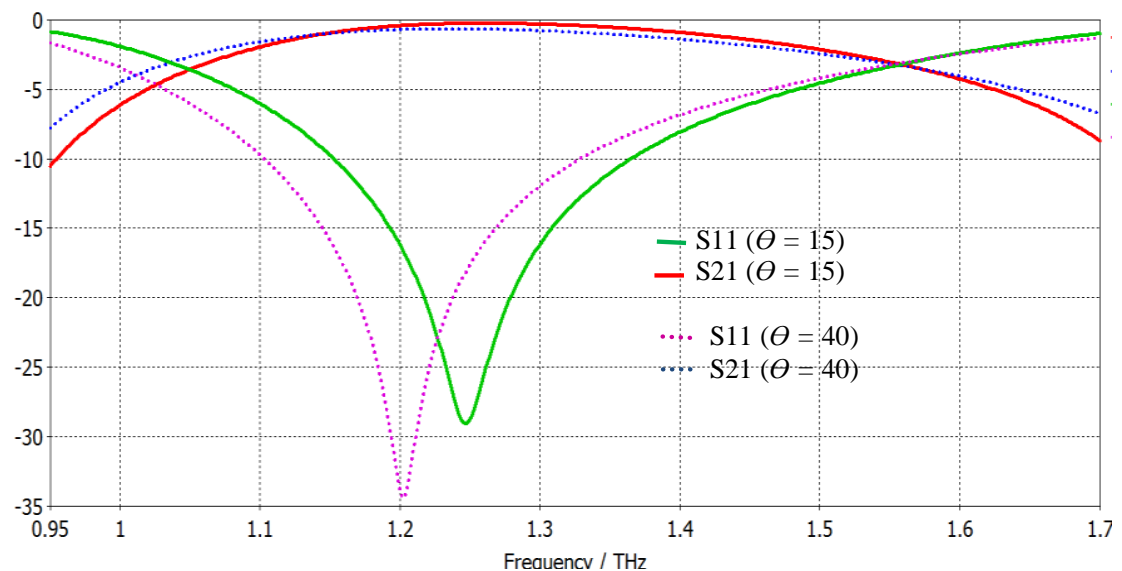

Fig 12. Transmission and reflection of a proposed bandpass filter with $\Theta=15^{\circ}$ and $40^{\circ}$ degree (simulate distribution structure in CST).

Table 1. Bandpass metal mesh filters from published work.

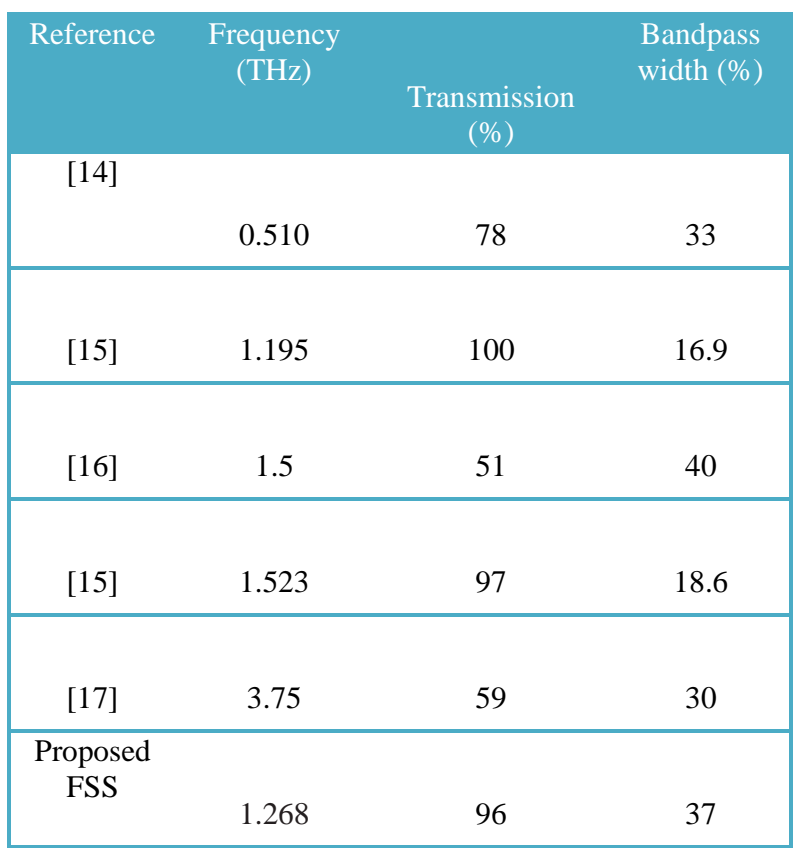

\section{Conclusion}

For the construction of $\mathrm{THz}$ filters, a unique structure is proposed. Due to its simple structure, which consists of a single metal layer and a dielectric layer, the suggested filter is relatively straightforward to manufacture. The proposed shape has been proved to have a $3 \mathrm{~dB}$ 
bandwidth of up to 45 percent and efficient band edge transition. As a single band FSS, this construction can be used. The counterpart of a clumped-LC-network is found. ADS was used to model the similar circuit. The response generated by the comparable circuit model is very similar to the FSS structure's response. The tuning of frequency resonators with constant fractional bandwidth can be achieved by changing the gap in the centre of the ring $\mathrm{G}_{\mathrm{c}}$. The study shows that the increase of the centre gap $\mathrm{G}_{\mathrm{c}}$ from $10 \mu \mathrm{m}$ to $14 \mu \mathrm{m}$ and $18 \mu \mathrm{m}$ can shift the resonant frequency from $1.18 \mathrm{THz}$ to $1.268 \mathrm{THz}$ and $1.31 \mathrm{THz}$ with gradually increasing in bandwidth. It is shown that the bandwidth can be increased when the incident angle is more than $0^{\circ}$ until 45 degrees, but at the same time the transmitted power continues to decrease for angle incident wave more than $0^{\circ}$. A comparison of the proposed structure result with bandpass metal mesh of different authors has been done.

\section{References}

[1] T. K. Nguyen, H. Han, I. Park "Highly Efficiency Resonant Antennas for Terahertz Photomixers", iWAT, 2012

[2] B. S. Rawat, A. Bhat, J. Pistora, "THz Band Nanoantennas for Future Mobile Communication", NY 89557-0260, U.S.A. 2013

[3] F. Costa, A. Monorchio, G. Manara, "Efficient Analysis of Frequency-Selective Surfaces by a Simple Equivalent-Circuit Model”, IEEE Antennas and Propagation Magazine, vol. 54, no. 4, 2012

[4] Lioubtchenko, A. V. Räisänen, and S. A. Tretyakov, "Simple and Accurate Analytical Model of Planar Grids and High-Impedance Surfaces Comprising Metal Strips or Patches," IEEE Transactions on Antennas and Propagation, AP-56, 6, 2008, pp. 1624-1632.

[5] R. Ulrich, "Far-Infrared Properties of Metallic Mesh and its Complementary Structure," Infrared Physics, 7, 1967, pp. 37-55

[6] L. Whitbourn, R. Compton, "Equivalent-circuit formulas for metal grid reflectors at a dielectric boundary", APPLIED OPTICS, Vol. 24, No. 2, 1985

[7] A. Melo, A. Gobbi, M. Piazzetta, A. da Silva, "Historical Review and Results Cross-Shaped Terahertz Metal Mesh Filters" Advances in Optical Technologies, Volume 2012

[8] Y. J. Chiang, T. J. Yen, "A high-transmission dualband terahertz bandpass filter by exciting multiresonance of metamaterials", Taiwan 2010

[9] N. Jin, J. Li, "Terahertz Wave Bandpass Filter Based on Metamaterials", Microwave and Optical Technology Letters, 2010

[10] L. Si, Y. Yuan, H. Sun, X. Lv, "Characterization and Application of Planar Terahertz Narrow bandpass Filter with Metamaterial Resonators", International Workshop on Metamaterials, 2008, China, 2008

[11] I. Al-Naib, Ch. Jansen, M. Koch, "Very Compact Bandpass Filter Based on Spiral Metamaterial Resonators", 34th International Conference on Infrared, Millimeter, and Terahertz Waves, 2009. IRMMW-THz 2009

[12] O. Paul, R. Beigang, M. Rahm,“Highly Selective Terahertz Bandpass Filters Based on Trapped Mode Excitation", OPTICS EXPRESS, 2009

[13] Y. Lee, "Principles of terahertz science and technology", 1st ed. New York, NY: Springer, 2008

[14] L. A. Page, E. S. Cheng, B. Golubovic, J. Gundersen, and S. S.Meyer, "Millimetersubmillimeter wavelength filter system," Applied Optics, vol. 33, no. 1, pp. 11-23, 1994.

[15] D. W. Porterfield, J. L.Hesler, R. Densing, E. R. Mueller, T. W. Crowe, and R. M. Weikle II, "Resonant metal-mesh bandpass filters for the far infrared," Applied Optics, vol. 33, no. 25, pp.6046-6052, 1994. 
[16] Kuznetsov, V. V. Kubarev, P. V. Kalinin et al., "Development and their application in highpower experiments at Novosibirsk of metal mesh based quasi-optical selective components $\mathrm{S}$. A. terahertz FEL," in Proceedings of the FEL, 2007.

[17] Y. Ma, A. Khalid, T. D. Drysdale, and D. R. S. Cumming, "Direct fabrication of terahertz optical devices on low-absorption polymer substrates," Optics Letters, vol. 34, no. 10, pp. 1555-1557, 2009. 